\title{
Téoros
}

Revue de recherche en tourisme

\section{Crise de la recherche, crise du tourisme}

\section{Jean Stafford}

Volume 1, numéro 2, 2e trimestre 1982

La recherche en tourisme : forces et faiblesses

URI : https://id.erudit.org/iderudit/1080853ar

DOI : https://doi.org/10.7202/1080853ar

Aller au sommaire du numéro

Éditeur(s)

Université du Québec à Montréal

ISSN

0712-8657 (imprimé)

1923-2705 (numérique)

Découvrir la revue

Citer cet article

Stafford, J. (1982). Crise de la recherche, crise du tourisme. Téoros, 1(2), 5-8.

https://doi.org/10.7202/1080853ar d'utilisation que vous pouvez consulter en ligne.

https://apropos.erudit.org/fr/usagers/politique-dutilisation/ 


\title{
Crise de la recherche, crise du tourisme
}

\author{
Par Jean Stafford
}

La science est une institution servant à produire des connaissances ou, utilisant un vocabulaire plus contemporain, un système secrétant de l'information. Dans cette perspective la recherche n'est qu'un mode de traitement spécialisé de l'information. La question centrale n'est pas de faire ou de ne pas faire des recherches mais de savoir si on peut vivre et prosperrer dans un système complexe comme la société actuelle sans information sur ce système?

En ce sens le tourisme au Québec est un cas type de système n'ayant pas ou peu d'information systématique (scientifique) sur lui-même; c'est une activité qui semble fonctionner tout seule sans que I'on connaisse les mécanismes de son fonctionnement!

Dans la plupart des congrès et des colloques portant sur le tourisme, on insiste lourdement sur la concertation entre les partenaires impliqués dans ce secteur; cette concertation sera impossible tant que les intervenants $n$ 'auront pas une bonne connaissance d'eux-mêmes et des différents environnements où ils sont appelés à travailler. Cette connaissance, cette information, seule une recherche systématique est en mesure de la fournir.

Cet article est consacré uniquement à l'analyse des causes qui limitent et freinent le développement des recherches sur le tourisme au Québec. Nous avons isolé trois types de causes pouvant favoriser les blocages face à la recherche scientifique dans le domaine du tourisme. Le premier type regroupe les causes conjoncturelles (1): ce sont celles qui dépendent directement de la crise économique et de la crise sociale que nous vivons actuellement. Le deuxième type touche surtout les causes tendancielles: elles font référence aux principaux acteursdécideurs du tourisme, à leurs comportements face à la recherche scientifique. Enfin, le troisième type isole les causes structurelles c'est-àdire les problèmes spécifiques que posent une véritable téorologie (ou science du tourisme).

Ces trois types de causes correspondent à des niveaux de complexité et d'abstraction différents; ils font aussi appel à une périodicité différente pour chacune des causes étudiées. Les causes conjoncturelles sont des variables exogènes donc plus faciles à saisir; elles sont aussi reliées à I'histoire socio-économique récente du Québec. Les causes tendancielles sont endogènes et supposent une tendance lourde au niveau des attitudes des acteurs-décideurs face à la recherche scientifique ces dernières années. Les causes structurelles sont inhérentes aux difficultés de formaliser une théorie cohérente du tourisme; c'est un problème à très long terme et il aura un impact important sur l'avenir de cette nouvelle discipline qu'est la téorologie. Ces trois types de causes interagissent ensemble et permettront d'expliquer. en tout ou en partie, les blocages actuels au niveau de la recherche en tourisme. En fonction de cette analyse nous tenterons d'indiquer les issues possibles à la crise de la recherche en tourisme au Québec.

\section{Les clauses conjoncturelles}

Depuis quelques années tous les indicateurs socio-économiques démontrent que nous sommes entrés dans une profonde récession. L'évolution de la conjoncture se mesure assez facilement en additionnant l'indice de chômage à l'indice d'inflation: ainsi pour le premier trimestre de 1982 l'indice de la crise est de 24.7 (il était de 25.6 en 1933 , la pire crise économique pour le Québec (2). La hausse vertigineuse des taux d'intérêt conforte cette récession et réduit la crise à un phénomène strictement monétaire (3).

Cette image est fausse: la crise actuelle est surtout le produit de facteurs multiples; selon Michel Godet: "Les crises résultent soit de l'évolution de tendances contradictoires (consommation, production selon le célèbre exemple de Malthus), soit de l'émergence d'une nouvelle tendance qui vient perturber l'équilibre en place (saut technologique par exemple)" (4). Toute crise suppose aussi une augmentation de l'incertitude générale, de la confusion et du désarroi à tous les niveaux de la structure sociale (politique, économique, social).

La crise actuelle peut aussi être l'indice d'un changement profond qui amènerait un approfondissement de la récession; cette récession correspondrait ainsi à une conjoncture d'assez longue période. Certains auteurs (5) expliquent cette récession en l'assimilant à la phase descendante (contraction) d'un cycle long dit de Kontradieff (du nom de son inventeur): le cycle long de Kondratieff dure une cinquantaine d'années et selon cette théorie:

- la période 1945-1970: l'expansion du cycle, donc une période de croissance et de relative prospérité:

- la période 1971-1995: contraction du cycle, décroissance, récession durant entre 20-25 années.

Cette conjoncture d'assez longue période peut être expliquée par un ensemble de facteurs:

- l'épuisement des gains de productivité dans tous les pays occidentaux;

- I'épuisement des différentes formes de consommation (achats alimentaires, achats de biens durables ou semi-durables);

- la croissance brutale des dépenses de I'État:

- la croissance du secteur tertiaire (les services) (6). La conjonction de ces quatre facteurs aboutit à une société restreinte où l'incertitude dominera, une société précaire qui réduira l'espace social et politique.

Si cette conjoncture se poursuit elle aura un impact négatif sur le développent du tourisme au Québec. 
Mais toute crise comporte aussi des éléments positifs. Un des aspects positifs de la crise est de montrer " ${ }^{*}$.que ce qui allait de soi, ce qui semblait fonctionnel, efficace, comporte des carences et des vices" (7). Elle indique qu'il y a une non-connaissance ou une méconnaissance des différents environnements. La crise peut aussi entraîner les décideurs, après le constat de non-fonctionnalité, à des attitudes régressives; ces attitudes vont amener, très souvent, une rigidité des rẻgles et normes anciennes; elles provoquent un blocage à tous les changements susceptibles d'améliorer la situation.

La limitation des recherches dans le domaine du tourisme au Québec est une conséquence directe de ces attitudes provoquées par la crise conjoncturelle qui sévit actuellement. Pour la plupart des organismes opérant dans le domaine du tourisme l'environnement devient plus opaque qu'auparavant. Cette opacité, due à la non-information, creuse un fossé de plus en plus large entre les décideurset l'évolution des marchés et des besoins des populations. Cette perte de contact va provoquer, à son tour, des crises organisationnelles (8). C'est un cercle vicieux: les causes conjoncturelles amènent une crise de la recherche: cette crise de la recherche va jouer un rôle important dans la crise actuelle du tourisme au Québec. Les décideurs doivent faire du "pilotage à vue" sans savoir réellement où ils vont!

\section{Les causes tendancielles}

Les clauses tendandielles vont siajouter aux causes conjoncturelles décrites plus haut et augmenter l'opacité entre le système touristique et ses différents environnements. Les causes tendancielles reposent, en grande partie, sur l'incapacité des différents niveaux de gouvernements et des organismes oeuvrant dans le champ du tourisme à évaluer correctement les coûts de "la noninformation: les uns et les autres font une mauvaise évaluation des effets de la non-connaissance vis-à-vis les besoins de l'ensemble de la population en matière de tourisme.

Cette évaluation des coûts peut se faire au niveau macro-économique et au niveau micro-économique. Au niveau macro-économique il s'agit de faire une comparaison qualitative et/ou quantitative entre les coûts de I'information (des recherches en tourisme) et les coûts de la noninformation (les coûts de l'inaction). Globalement on peut déterminer cing types de coutts (9):

1. C.R.: coûts de la recherche ellemême (salaires, dépenses diverses. etc...);

2. C.C.: coûts de la consommation de cette information (pour répondre, expliquer, sensibiliser les gens aux résultats des recherche: ce sont les coûts de la "digestion de I'information" "):

3. C.A.: coûts des actions à mener en fonction des résultats de ces recherches (politiques, programmes, projets à mettre en oeuvre, etc...):

4. C.C.V.: coûts des conflits de valeurs ou des changements qu'amènent ces informations (changements d'objectifs, changements organisationnels, changements dans les statuts, les róles et les modèles de comportements, etc...);

5. C.I.: les coûts que pourraient provoquer l'inaction (ce qui arriverait si on ne fait rien, si on "laisse aller" les choses): par exemple le Conseil économique du Canada dans son Dix-huitième Exposé annuel évalue le coût de l'inaction, au point de vue des politiques économiques, d'ici 1990 à 91 milliards de dollars (10):

On va appeler U.G. I'utilité globale de I"information obtenue par le biais des recherches en tourisme. On peut penser, que pour les différents paliers de gouvernement et les organismes, les recherches seront faites et utilisées quand les coûts de I'inaction et de la non-information dépasseront les coûts d'implantation (soit les autres coúts) dans cette perspective l'équation de I'évaluation des coûts prendra la forme suivante:

U.G. $\geq$ C.R. + C.C. + C.A. + C.C.V. - C.I.

Dans le cas contraire si l'inaction semble moins couteuse que l'action on aura l'équation suivante:

UG $\leq$ C.R. + C.C. + C.A. + C.C.V. - C.I.

Ici il ne faut pas sous-estimer I'inertie habituelle des différentes administrations; chacune d'entre elles est le produit d'une longue histoire qui a favorisé le développement d'idiosyncrasies qui limitent très souvent les possibilités de changement et I'adoption d'approches et de méthodes nouvelles.

La principale difficulté de ce modèle d'évaluation des coûts de l'inaction est d'apprécier correctement certains types de coûts: par exemple l'analyse des conflits de valeurs en des termes monétaires peut poser certains problèmes concrets de définition mais ce sont les mêmes difficultés que l'on rencontre habituellement dans l'établissement d'une comptabilité sociale (11).

Au niveau micro-économique l'évaluation des coûts de l'inaction passe par une analyse avantages-coûts de chacune des recherches en tourisme. Cette analyse doit pouvoir mesurer la rentabilité de ces recherches en prenant en considération (d'une façon systématique) les dimensions internes et les dimensions externes propres à toute recherche et en dégageant les avantages et les inconvénients d'un point de vue monétaire et non-monétaire. Ce mode d'évaluation peut être schérnatisé de la façon suivante (12):

\begin{tabular}{|c|c|c|c|c|}
\hline \multirow[b]{2}{*}{$\begin{array}{l}\text { Eláments } \\
\text { monitaires }\end{array}$} & \multicolumn{2}{|c|}{ Valeur interne al l'organisme } & \multicolumn{2}{|c|}{ Valeur externe a l'organisme (valeur sociale) } \\
\hline & $\begin{array}{l}\text { Avantages } \\
\text { - Meilleure connaissance } \\
\text { du marché } \\
\text { - Meilleures décisions } \\
\text { - Meilleure planification }\end{array}$ & $\begin{array}{l}\text { Ineonvánients } \\
\text { - Coût global de la } \\
\text { recherche } \\
\text { - Couts en temps } \\
\text { - Coúts en surcharge de } \\
\text { personnel } \\
\text { - Augmentetion générale } \\
\text { des couts dioperation }\end{array}$ & $\begin{array}{l}\text { Avantages } \\
\text { - Effets d antrainement } \\
\text { sur d autres secteurs lies } \\
\text { au produit gu au service } \\
\text { - Pecettes induites par } \\
\text { une meilleurs imagu } \\
\text { extéfieure }\end{array}$ & $\begin{array}{l}\text { Ineonvenients } \\
\text { - Plus grande competition } \\
\text { entre arganismes } \\
\text { - Monopole de } \\
\text { linformation }\end{array}$ \\
\hline $\begin{array}{l}\text { Eléments } \\
\text { non monétaires }\end{array}$ & $\begin{array}{l}\text { - Rationalite } \\
\text { - Meilleure connaissance } \\
\text { des ebjectifs } \\
\text { - Demarche a mơyen terme } \\
\text { et à long terme } \\
\text { - Plus grande implication } \\
\text { du personnel }\end{array}$ & $\begin{array}{l}\text { - Caractère } \\
\text { "réductioniniste" des } \\
\text { recherches. } \\
\text { - Changement dans les } \\
\text { habitudes de travail } \\
\text { " "Froideur" } \\
\text { crganisationnelle }\end{array}$ & $\begin{array}{l}\text { - Meilleure accassibilate } \\
\text { du service oroduit } \\
\text { - Meilleure image a } \\
\text { l'exterrieur au plan } \\
\text { profossionnel }\end{array}$ & $\begin{array}{l}\text { - Abandion de certains } \\
\text { objectifs jugiss peu } \\
\text { reniables } \\
\text { - Elimination du } \\
\text { benenvolat et de la } \\
\text { gratuite }\end{array}$ \\
\hline
\end{tabular}


Ce schéma tient compte de tous les aspects entrant dans l'évaluation d'une recherche. Si les inconvenients monétaires et non monétaires, internes et externes, dépassent les avantages monétaires et non monétaires, internes et externes, l'organisme (la firme ou le service gouvernemental) n "a pas intérêt à effectuer des recherches en matière de tourisme. Dans le cas ou les avantages dépassent les inconvénients l'organisme a un intérêt réel à avoir accès à l'information souhaitée (les coûts de l'inaction dépassent les coûts de I'action).

L'impuissance tendancielle à évaluer valablement les coûts de la noninformation scientifique va avoir un impact négatif important sur l'ensemble du système touristique québécois. Dans ce système, où l'on peut plus facilement parler de "désinformation" que d'information, la concertation des acteurs, si étroitement reliée au succès de toute politique touristique, dépend aussi de la capacité de produire de linformation scientifique sur ce milieu. Dans ce systeme quatre situation sont possibles:

Production d'information scientifique en tourisme et concertation des agents du système touristique.

\begin{tabular}{|c|c|c|c|}
\hline & \multicolumn{2}{|c|}{ Production d'information } \\
\hline & & Forte: & Faible \\
\hline 5 & Forte & 1 & 2 \\
\hline$\dot{0}$ & Faible & 3 & 4 \\
\hline
\end{tabular}

Dans cette typologie la situation 1 correspond $a$ un ajustement mutuel entre les agents, la production forte d'information scientifique va favoriser cette concertation maximum. Dans la situation 2 la concertation reste forte même si la production d'information scientifique est faible, cela suppose qu'une "idéologie dominante" sert de ciment et oriente l'ensemble des acteurs dans ce domaine, La situation 3 signifie que chacun des organismes tient à conserver ses propres informations; cette situation suppose une forte concurrence entre les organismes et un monopole exercé par certains d'entre eux. Enfin, la $4 e$ situation représente ce qui se passe actuellement au Québec: production d'information scientifique faible et concertation faible: c'est le degré zéro, le retrait sur soi!

\section{Les causes structurelles}

Les causes structurelles du manque actuel de recherche scientifique en tourisme apparaissent dans les difficultés d'établir une véritable "science du tourisme" (une téorologie): ces difficultés sont considerables et elles limitent, et limiteront pour longtemps, la structuration de cette nouvelle discipline! Ces causes structurelles sont des problèmes épistémologiques spécifiques a toute démarche scientifique.

La meilleure façon de cerner ces causes structurelles. ces problèmes épistémologiques, c'est de répondre a la question: c'est quoi une science? Une science c'est, avant tout, des chercheurs qui formulent des hypothèses sur la réalité étudiée dans un domaine donné et tentent, avec des instruments de recherche et d'analyse. d'infirmer ou de confirmer ces hypotheses. Ces chercheurs travaillent à l'intérieur d'un cadre de pensée, d'un courant d'idées, qu'on appelle un paradigme: les membres de ce paradigme possèdent les mêmes valeurs sociales et les mêmes orientations théoriques et méthodologiques et luttent en rangs serrés contre les autres paradigmes à l'intérieur d'une même discipline.

Le paradigme va déterminer les questions jugées importantes pour un groupe de chercheurs et déterminer aussi les movens de les étudier (13). La puissance de la méthode scientifique tient dans sa capacité de formuler des paradigmes.

"La puissance de la méthode scientifique tient essentiellement au fait que l'esprit scientifique accepte un paradigme et par consequent limite son champ d'investigation. A partir du moment oú Fon accepte un point de vue restreint, commande par des theories, on peut determiner les problèmes que l'on veut résoudre ety concentrer toutes ses énergies. Auparavant, on faisait face a un ensemble de questions si mal definies quill devenait impossible d'y voir clair" $(14)$.

Le paradigme va ainsi permettre une délimitation stricte de l'objet d'étude; celui-ci sera le résultat: "D'un processus continuel de rupture avec les prénotions du sens commun, avec les connaissances vagues, mythiques ou idéologiques" (15). Un autre élément important d'un paradigme est de permettre, selon l'expression de Karl Popper, la falsification des hypothèses (16). Cette falsification est une exigence fondamentale de toute demarche scientifique; selon cette exigence: "Seules à revetir un caractère scientifique seront les propositions qui peuvent être placées sous des conditions telles qu'elles risquent d'être rejetées par l'épreuve" (17). Dans cette perspective une hypothèse scientifique est une hypothese dont il est possible de vérifier la faussetél Sans paradigme, il n'y a pas de science possible.

La principale cause structurelle de l'absence de recherche fondamentale en tourisme est que la téorologie lou la science du tourismel est préparadigmatique. II $\mathrm{y} a$, bien sûr, des recherches qui se font en tourisme mais la plupart d'entre elles sont axées sur des résultats concrets et immédiats; ce sont des démarches ponctuelles orientées vers des solutions pratiques; ces approches du phénomène touristique sont nécessaires mais non suffisantes pour former un ou plusieurs paradigmes.

Le tourisme n'est pas un objet scientifiquement défini à travers une démarche théorique et méthodologique; il n'y a pas de consensus sur les questions vitales pour le développement d'une science du tourisme. Les seules approches valables dans ce domaine sont des recherches sectorielles qui se font par le biais des études de marketing (18), de sociologie (19) et d'économie (20) du tourisme. Au plan formel certains efforts de systématisation ont été faits mais ce sont surtout des essais de francstireurs de la recherche en tourisme et non de groupes de recherche organisés (21).

Le caractère non marchand du tourisme est surtout représenté par les tenants du tourisme social; ce courant de pensée est surtout basé sur une certaine conception de l'éthique sociale. Actuellement le tourisme social est beaucoup plus une idéologie qu'un paradigme scientifique; il véhicule les mêmes erreurs épistémologiques et les mêmes faiblesses théoriques et méthodologiques que la sociologie du loisir défendue naguère par Joffre Dumazedier (22). Une dimension critique apparaît aussi dans l'étude du développement du tourisme dans les pays en voie de développement (23) mais il en faut plus pour constituer un paradigme.

Le caractère pré-paradigmatique de la téorologie est un obstacle important au développement de cette nouvelle discipline: il limite la capacité de cerner les problèmes importants dans ce domaine et réduit d'autant la possibilité de définir d'une façon spécifique l'objet d'étude du tourisme. Le tourisme, sans des paradigmes structurés, demeurera un phénomène résiduel, à jamais insaisissable scientifiquement et toujours à la remorque des autres sciences sociales. 


\section{Pour sortir de la crise}

Les causes que nous avons décrites et analysées contribuent à réduire le nombre des recherches et amènent une perte considérable d'information pertinente sur le milieu touristique québécois: celui-ci devient une zone d'ombre ou prédomine le pilotage à vue alors que nos concurrents, les états voisins, disposent des informations nécessaires au développement de leur industrie touristique.

Au niveau global, ces causes s'additionnent et expliquent le degré zéro de la recherche en tourisme au Québec. Au niveau de l'analyse, chacune de ces causes du manque de recherche en tourisme est différente et exige des solutions différentes!

On peut diminuer l'influence des causes conjoncturelles en utilisant les armes habituelles de la lutte anticyclique. Sans tomber dans un keynesianisme à rabais, en faisant certains investissements, it serait possible de rendre attrayantes les destinations québécoises pour revitaliserle tourisme. Une multitude de programmes, articulés les uns aux autres, pourrait favoriser une reprise économique dans ce secteur; ces programmes pourraient être définis à partir de la recherche des marchés, des attitudes et des comportements des touristes. En subventionnant la recherche pour les organismes intéressés le gouvernement du Québec ne ferait que remplir son rôle face à une industrie importante, laissée à ellemême et de plus en plus menacee.

La lutte contre les causes conjoncturelles doit passer par une compréhension de la complexité du phénomène touristique: il faudrait s'adapter à cette complexité au lieu de régresser vers des règles et des méthodes toutes faites. Face au désarroi, à l'indécision qu'entraine la crise actuelle il faudrait proposer de nouvelles orientations, de nouvelles approches: c'est le moment d'inventer et/ou d'adopter de nouvelles formes de gestion et d'animation dans le monde du tourisme. La philosophie de la "société restreinte" doit être rejetée au profit d'une société ouverte, axée sur la connaissance et I"analyse des différents environnements.

La réduction des causes tendancielles dépend de l'évolution des mentalités des acteurs-décideurs du milieu du tourisme; ceux-ci se doivent de privilégier une gestion de l'information centrée sur la connaissance scientifique des différents éléments du système touristique québécois. II faut sensibiliser et convaincre les acteursdécideurs de l'importance cruciale d'une information pertinente et des coûts de la non-information face aux différents environnements.

Cette entreprise de sensibilisation doit se faire:

1. par un accroissement des liensentre les chercheurs et les acteursdécideurs par le biais du Centre d'études en tourisme:

2. par la diffusion et la socialisation des informations, c'est-à-dire s'assurer que les informations soient connues. comprises, assimilées et débouchent sur des décisions et des actions;

3. par une formation continue des acteurs-décideurs, formation orientée vers la gestion de l'information et les coûts de la noninformation.

II serait illusoire d'espérer apporter rapidement des solutions aux causes structurelles du manque de recherche en tourisme au Québec. Le caractère pré-paradigmatique de la téorologie va limiter pendant longtemps le développement de cette nouvelle science du tourisme

Les seules solutions valables reposent sur la critique et l'approfondissement des embryons de paradigmes qui existent déjà: cette critique est essentielle car, selon le grand épistémologue Gaston Bachelard: "La connaissance cohérente est un produit non pas de la raison architectonique, mais de la raison polémique" (24).

L'analyse des causes conjoncturelles, tendancielles et structurelles du manque de recherche en tourisme nous donne un tableau d'ensemble des obstacles à une connaissance structurée dans ce domaine. Pour apporter des solutions il faut percevoir ces obstacles d'une façon dynamique. Les causes conjoncturelles sont limitées dans le temps, elles supposent des réponses immédiates et concrètes.

Les causes tendancielles dépendent de l'évolution des attitudes des acteursdécideurs face à la recherche, ces attitudes ne sont pas immuables et pourraient changer rapidement si des efforts soutenus sont faits dans ce sens. Les solutions aux causes structurelles exigeront un très long travail d'élaboration théorique et méthodologique.

II ne faut pas désespérer, on peut croire qu'à très long terme de nouveaux paradigmes du tourisme pourraient émerger à partir d'une théorie générale du temps et d'une théorie générale de l'espace (environnement). Temps et espace intimement liés seraient alors les deux axes d'une science du tourisme de l'avenir.

(1) Voir au sujet de ces concepts: Chrishian GOUX, L'horizon prévisionnel, Editions Cujas, Paris, 1969

( 2) Voir: DUBuC, A, L'indice de ta misere est plus dilewe oue dans les annies 30 , in La Presse, samedi 23 janvier 1982

( 3) Voir: BOULEY, G., Pour whe monnaie qui inspire cowhance ou les exigences of une prosperite save inflation. in Le Devoir, jeudi le 25 mars 1982

( 4) GODET, M. Demain les crises. De la résignation a I'entifatalite, Hacherte. Paris. 1980. p. 16.

(5) Voir a ce sujet:

- MANDEL, E. La crise 1974-1978, Flammarion, coll. Champs, Paris, 1978

- KAHN. H. World Economic Development Morrow Ouill Paperback, N.Y, 1979, pp. 208-216

- AOSTOW. WW. L'ultimatum de l'an 2000 , Economica, Paris, 1978.

( 6) LORENZI, J.H., PASTRE, O. TOLEQANO, J. La cris du XXe siecle, Economica, Paris 1980 po. 223-274.

( 7$)$ MORIN, E. Pour une erisologie, in Communications. กอ 24 . Sevil, Paris, 1976, p. 159

( B) Voir: SMART, C.F. STANBURY, W.T et all, Study on Crisis Management, Institute far Reserch on Publis Policy. Montréal, 1978

(9) A ce sujet voir les travaux de R. AOSE: The market for policy indocators in Social Indicators and Social Policy. Heinemann, London, 1972, pp. 124-127.

(10) Sortir de l'impasse, Conseil éconemique du Canads Dix-huitieme Expose annuel, Ottawwa, 1981, p. 67

(11) Voir à ce sujet

- Ecrit en collaboration Mesure du bien-etre social. Progres accomplis dans I'elaboration d'indicateurs sociaux O.C.D.E., Paris. 1976 .

- ZAPF. W. Sacial indicators Praspects for Socia Accounting Systems in Social Sciences Information, Aussell Sage Foundation, New York. 1972

(12) Nous nous inspirans ici de la demarche de Jéröm BON et Albert LOUPPE Marketing des services publics: l'etude des bessoins de la population, Les editions do organisation, Paris, 1980, p. 110

(13) Voir at ce sujet: KUHN, T.S., La structure des revolu tions scientifiques, Flammarion, Paris, 1972.

(14) FOUREZ, G. La science partisane. Duculot, Gembloux, 1974. p. 76

(15) BRUYNE. P.. HEAMAN, J. SCHOUTHEETE, J. DYnamique de la recherche en sciences sociales, P.U.F. Paris, 1974. p. 48.

(16) POPPER, K., La logique de la découverte scientifique. Payot, Paris, 1973, chapitre III

(17INALIMOV, V., les struciures de la science et $\mathrm{f}$ réceptivie des hyouheses. in Diogène. no 100. Gallimard, Paris, 1977, a. 202

(18) Par exemple woir:

- fillatreault. P. Ritchie, B., Family Vocodia Decisian Making. A Aleplicatian and Exferision, in Joumal of Travel Research, Vol XVIII, no 4. 1990

- ARSENAULT. J. FORTIN, P., AITCHIE, d. Une étude processus décisionnel des associations nord. americaines dans le choix d"un site de congras e le comportement d'achat des organisations. Wot con polto duchat des organisations, Vol planificarion et de developperment du Queber. planification

(19) Par exemple l'apgroche ethnométhodologique de MAC CANNELL. D. The Teurist, Sehacken Books, MY. 1976

$120)$ Voir le classique du genre: DA.AET,JE, R. DEFERT, P. Aspeets teonomiques du tourisme. Berger-Levrmault, Paris, 1972

(21) Voir a titre dexemples:

- RENOUX M. Techniques feconométriques de prtvision de la demande touristique et amorce de eurs integrations dans un systeme decisionnel. Gouvernement du Quthec, Quebec, 1972

- LAINE, P. Libenons le tourisme. Editions Favolle. Paris 1980 .

(22) Voir la critiqué pénétrante et définitive de la sociologis dumardienne dians: LANFANT. M.F. Les thefories de loisir, P.U.F., Paris, 1972

(23) Voir: ROZEMBERG, D. et al., Towrisme intemationale societes locoles in Problemes politiques at sociaux. no 423. La Documentation Française. Paris. septembre 1981.

(24) BACHELARD, G., La philosophie du non. PU.F., Paris. 1962. p. 139 